\title{
A GROWTH MIXTURE THEORY FOR CARTILAGE
}

\author{
Stephen M. Klisch ${ }^{1,2}$, Robert L. Sah', and Anne Hoger ${ }^{1}$ \\ ${ }^{1}$ Department of Mechanical and Aerospace Engineering \\ 2Department of Bioengineering \\ University of California, San Diego \\ La Jolla, CA
}

Mechanics in Biology, Editors. J Casey and G Bao, ASME AMD \& BED (Vol. 242), pp. 229-242, New

York, 2000.

\section{ABSTRACT}

In this paper we present a model of growth for cartilaginous tissues in which there exists a saturated solid matrix composed of multiple constituents that may grow and remodel independently of each other. Klisch and Hoger recently developed a general theory of volumetric growth for a mixture of $v-1$ growing elastic materials and an inviscid fluid, which included a treatment of two special types of internal constraints that are relevant to cartilage. Here, that theory is specialized to construct a cartilage growth model. This theory allows the constituents of the solid matrix to grow independently of each other, and can model the evolution of the constituent pre-stresses and the tissue's mechanical properties during developmental growth and degeneration. A simple example is presented which illustrates these features of the theory.

\section{INTRODUCTION}

Cartilaginous tissues are composed primarily of three constituents: water, collagen, and proteoglycans. The results of numerous studies suggest that mechanical stimuli cause growth abnormalities in conditions such as hip dysplasia (Pauwels, 1976), traumatic injury near the growth plate (Ogden, 1988), and scoliosis of the spine (Brickley-Parsons and Glimcher, 1984). There also exists experimental evidence from in vivo (Kiviranta et al., 1994) and in vitro (Sah et al., 1989) experiments that growth-related parameters such as biochemical composition, mechanical properties, and cartilage thickness vary due to changes in mechanical stimuli such as stress and strain. In addition, these studies suggest that the proteoglycan and collagen constituents of the solid matrix may grow and remodel independently of each other.

Continuum mixture theory has been used to model the finite deformation and flow-dependent mechanical properties of both articular cartilage (Ateshian et al., 1997) and the intervertebral disc (Klisch and Lotz, 2000). In those theories, the mixture is modeled as being composed of a solid constituent representing the collagen-proteoglycan matrix and a fluid constituent representing the water. As a first step towards developing a model of growth and remodeling for cartilaginous tissues, a general theory of volumetric growth for compressible elastic materials was presented by Klisch et al. (2000). That paper 
extended the work of Rodriguez et al. (1994) and Hoger (2000), who only considered incompressible materials. In those papers, the material added during growth was assumed to have the same mechanical properties as the original material. In addition, the deformation gradient due to growth was decomposed into two parts: a growth tensor that describes the amount and orientation of mass deposition, and an elastic accomodation tensor that ensures compatibility of the grown body. Furthermore, the stress in the material was independent of the growth tensor. The theory has recently been extended to thermoelastic materials (Klisch and Hoger, 2000a) and to a mixture of an arbitrary number of thermoelastic materials and an inviscid fluid (Klisch and Hoger, 2000b).

In this paper, we specialize this growth mixture theory to develop a model of growth for cartilaginous tissues. First, we summarize the theory of volumetric growth for a mixture of an arbitrary number of growing elastic materials and an inviscid fluid. Then, the theory is specialized to construct a cartilage growth model. This theory allows the constituents of the solid matrix to grow independently of each other, and is capable of modeling the evolution of the constituent concentrations, the constituent pre-stresses, and the tissue's mechanical properties. A simple example is presented which illustrates these features of the theory.

\section{PRELIMINARIES}

Consider a mixture B of $v$ constituents $^{1}: v-1$ growing elastic materials $C^{\alpha}(\alpha=1, v-1)$ and an inviscid fluid $C^{v}$; this will be referred to as a growing mixture. Let $\kappa_{0}(\mathrm{~B})$ denote a fixed, unloaded reference configuration of the mixture and $\kappa(B)$ denote the time-dependent loaded configuration of the mixture during a continuous growth process. A material particle of $C^{\alpha}$ occupies position $\mathbf{X}^{\alpha}$ in $\kappa_{0}(\mathrm{~B})$ and position $\mathbf{x}^{\alpha}$ at time $\mathrm{t}$ in $\kappa(\mathrm{B})$. In the configuration $\kappa(\mathrm{B})$ at time $\mathrm{t}$, it is assumed that there exists one material point of each $C^{\alpha}$ at every point $\mathbf{x}$ in the mixture such that $\mathbf{x}=\mathbf{x}^{\alpha}(\alpha=1, v)$. For simplicity, we will assume that $C^{\alpha}$ is free of residual stress in $\kappa_{0}(\mathrm{~B})^{2}$. It is assumed that the set of material points of $C^{\alpha}$ in the reference configuration $\kappa_{0}(\mathrm{~B})$ is dense in the set of all points in the present configuration $\kappa(\mathrm{B})$. The motion of $C^{\alpha}$ and the common temperature of the mixture are defined by sufficiently smooth mappings

$$
\mathbf{x}^{\alpha}=\chi^{\alpha}\left(\mathbf{X}^{\alpha}, \mathrm{t}\right), \quad \theta=\Theta\left(\mathbf{X}^{\alpha}, t\right)
$$

respectively, where $\theta>0$ is the absolute temperature of the mixture.

The density of $C^{\alpha}$ is $\rho^{\alpha}$ and the density of the mixture is defined as

$$
\rho=\sum_{\alpha=1}^{v} \rho^{\alpha}
$$

The velocity of $C^{\alpha}$ is

$$
\mathbf{v}^{\alpha}=\frac{\mathrm{d}^{\alpha}}{\mathrm{dt}} \chi^{\alpha}\left(\mathbf{X}^{\alpha}, \mathrm{t}\right)
$$

where the material time derivative $\mathrm{d}^{\alpha}(\cdot) / \mathrm{dt}$ follows the motion of $C^{\alpha}$. Relative velocities $\mathbf{a}^{\alpha}$ are defined as

$$
\mathbf{a}^{\alpha}=\mathbf{v}^{\alpha}-\mathbf{v}^{v}
$$

\footnotetext{
${ }^{1}$ Our notation follows that of Craine et al. (1970) who developed a mixture theory for an arbitrary number of viscous elastic materials.

${ }^{2}$ See Klisch et al. (2000) for how the theory may be modified to include a residual stress field in the reference configuration $\kappa_{0}(\mathrm{~B})$.
} 
The deformation gradient $\mathbf{F}^{\alpha}$ of the mapping from $\kappa_{0}(\mathrm{~B})$ to $\kappa(\mathrm{B})$ for each growing elastic material $C^{\alpha}$ is assumed to obey the decomposition ${ }^{3}$ (Figure 1)

$$
\mathbf{F}^{\alpha}=\mathbf{F}_{l}^{\alpha} \mathbf{M}_{e}^{\alpha} \mathbf{M}_{g}^{\alpha},(\alpha=1, v-1) .
$$

The tensor $\mathbf{M}_{e}^{\alpha} \mathbf{M}_{g}^{\alpha}$ describes the deformation due to growth of $C^{\alpha}$ relative to $\kappa_{0}(\mathrm{~B})$, whereas the deformation gradient $\mathbf{F}_{l}^{\alpha}$ represents a superposed elastic deformation of $C^{\alpha}$ caused by applied loads. It was established by Klisch et al. (2000) that the amount and orientation of mass deposition are described by $\mathbf{M}_{g}^{\alpha}$ and that the mass and free energy density functions are independent of $\mathbf{M}_{g}^{\alpha}$.

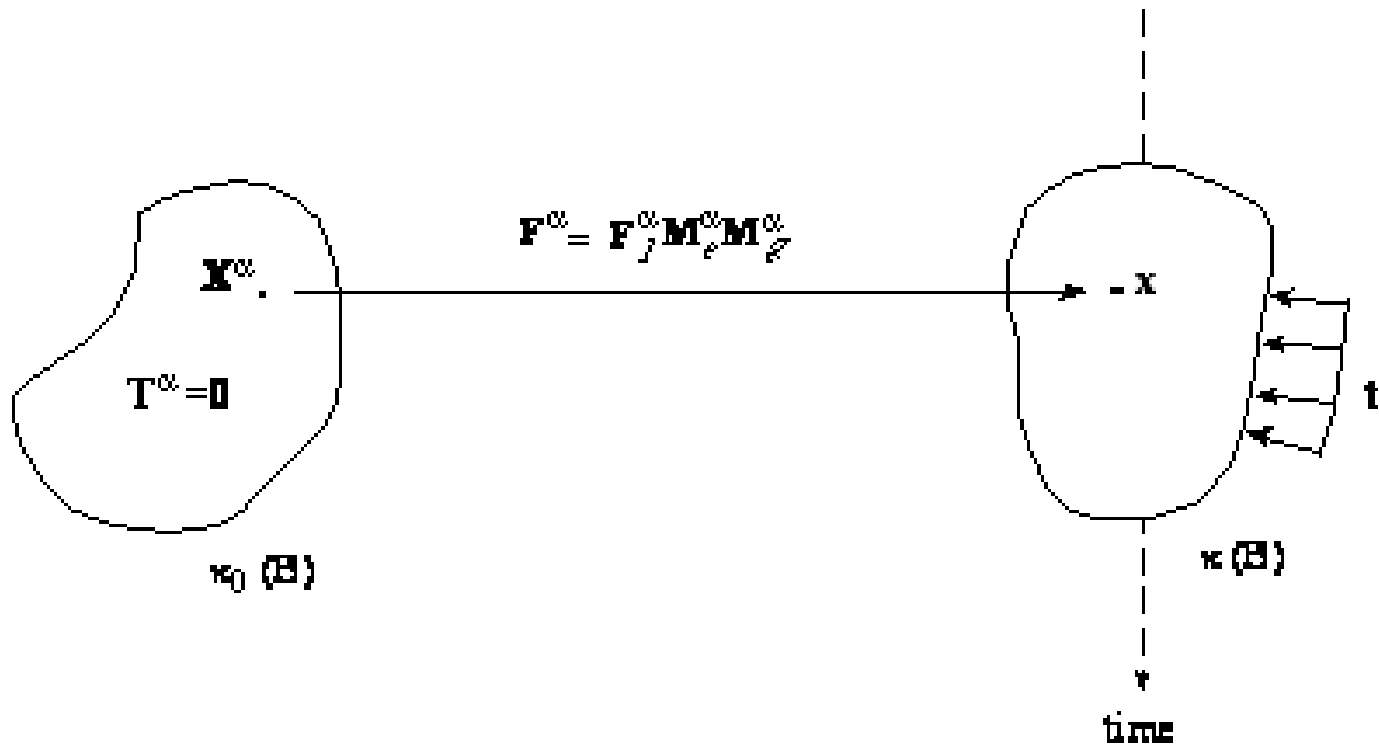

Figure 1: Schematic of the deformations in the growth mixture theory.

With the definitions

$$
\mathbf{L}_{l}^{\alpha}=\frac{\mathrm{d}^{\alpha} \mathbf{F}_{l}^{\alpha}}{\mathrm{dt}} \mathbf{F}_{l}^{\alpha^{-1}}, \quad \mathbf{L}_{e}^{\alpha}=\frac{\mathrm{d}^{\alpha} \mathbf{M}_{e}^{\alpha}}{\mathrm{dt}} \mathbf{M}_{e}^{\alpha^{-1}}, \quad \mathbf{L}_{g}^{\alpha}=\frac{\mathrm{d}^{\alpha} \mathbf{M}_{g}^{\alpha}}{\mathrm{dt}} \mathbf{M}_{g}^{\alpha^{-1}}, \quad(\alpha=1, \nu-1),
$$

the velocity gradient tensor derived from $\mathbf{F}^{\alpha}$ is

$$
\mathbf{L}^{\alpha}=\frac{\mathrm{d}^{\alpha} \mathbf{F}^{\alpha}}{\mathrm{dt}} \mathbf{F}^{\alpha^{-1}}=\mathbf{L}_{l}^{\alpha}+\mathbf{F}_{l}^{\alpha} \mathbf{L}_{e}^{\alpha} \mathbf{F}_{l}^{\alpha^{-1}}+\mathbf{F}_{l}^{\alpha} \mathbf{M}_{e}^{\alpha} \mathbf{L}_{g}^{\alpha} \mathbf{M}_{e}^{\alpha^{-1}} \mathbf{F}_{l}^{\alpha^{-1}},(\alpha=1, v-1) .
$$

\footnotetext{
${ }^{3}$ Since we are using a fixed reference configuration, the tensors $\mathbf{M}_{g}^{\alpha}$ and $\mathbf{M}_{e}^{\alpha}$ are not equivalent to the incremental growth and elastic accommodation tensors of Hoger (2000).
} 
The symmetric parts of $\left(\mathbf{L}_{l}^{\alpha}, \mathbf{L}_{e}^{\alpha}, \mathbf{L}_{g}^{\alpha}\right)$ are denoted as $\left(\mathbf{D}_{l}^{\alpha}, \mathbf{D}_{e}^{\alpha}, \mathbf{D}_{g}^{\alpha}\right)$, respectively. Using standard results, one can obtain

$$
\frac{1}{\mathbf{J}^{\alpha}} \frac{\mathrm{d}^{\alpha} \mathbf{J}^{\alpha}}{\mathrm{dt}}=\mathbf{D}^{\alpha} \cdot \mathbf{1}, \quad \frac{1}{\operatorname{det} \mathbf{M}_{g}^{\alpha}} \frac{\mathrm{d}^{\alpha} \operatorname{det} \mathbf{M}_{g}^{\alpha}}{\mathrm{dt}}=\mathbf{D}_{g}^{\alpha} \cdot \mathbf{1}, \quad(\alpha=1, v-1)
$$

The effective elastic deformation $\mathbf{F}_{*}^{\alpha}$ is defined as

$$
\mathbf{F}_{*}^{\alpha}=\mathbf{F}_{l}^{\alpha} \mathbf{M}_{e}^{\alpha},(\alpha=1, v-1) .
$$

The balance laws for a growing mixture include variables associated with mass deposition and growth energy supply that do not appear in the traditional balance laws. In particular, the balance laws for mass, linear momentum, angular momentum, and energy are

$$
\begin{gathered}
\frac{d^{\alpha} \rho^{\alpha}}{d t}+\rho^{\alpha} \operatorname{div} \mathbf{v}^{\alpha}=\rho^{\alpha} c^{\alpha},(\alpha=1, v-1), \quad \frac{d^{v} \rho^{v}}{d t}+\rho^{v} \operatorname{div} \mathbf{v}^{v}=0 ; \\
\rho^{\alpha} \frac{d^{\alpha} \mathbf{v}^{\alpha}}{d t}=\operatorname{div} \mathbf{T}^{\alpha}+\pi^{\alpha}+\rho^{\alpha} \mathbf{b}^{\alpha} ; \\
\rho^{\alpha} \frac{d^{\alpha} \varepsilon^{\alpha}}{d t}=\rho^{\alpha} r^{\alpha}-\mathbf{T}^{\alpha^{T}}=\Lambda^{\alpha} ; \\
\rho^{v} \frac{d^{v} \varepsilon \mathbf{q}^{\alpha}+\gamma^{\alpha}+\rho^{\alpha} \beta^{\alpha}+\mathbf{T}^{\alpha} \cdot \mathbf{D}^{\alpha},(\alpha=1, v-1),}{d t}=\rho^{v} r^{v}-\operatorname{divq}^{v}+\gamma^{v}+\mathbf{T}^{v} \cdot \mathbf{D}^{v} ;
\end{gathered}
$$

where $\mathrm{c}^{\alpha}$ is the mass growth function (i.e., the rate of mass deposition per unit current mass), $\mathbf{T}^{\alpha}$ is the partial Cauchy stress tensor, $\pi^{\alpha}$ is the diffusive force, $\mathbf{b}^{\alpha}$ is the partial external body force, $\Lambda^{\alpha}$ is the internal body couple, $\varepsilon^{\alpha}$ is the partial internal energy, $r^{\alpha}$ is the partial external heat supply, $\mathbf{q}^{\alpha}$ is the partial heat flux, $\gamma^{\alpha}$ is the internal energy supply, and $\beta^{\alpha}$ is the growth energy density supplied by an external source for $C^{\alpha}$. In addition, we postulate the growth continuity equation

$$
\operatorname{det} \mathbf{M}_{g}^{\alpha}=\exp \left[\int_{\tau=\mathrm{t} 0}^{\mathrm{t}} \mathrm{c}^{\alpha} \mathrm{d} \tau\right],(\alpha=1, v-1)
$$

or, equivalently,

$$
\mathrm{c}^{\alpha}=\frac{1}{\operatorname{det} \mathbf{M}_{g}^{\alpha}} \frac{\mathrm{d}^{\alpha} \operatorname{det} \mathbf{M}_{g}^{\alpha}}{\mathrm{dt}}=\mathbf{D}_{g}^{\alpha} \cdot \mathbf{1}, \quad(\alpha=1, v-1) .
$$

Here, $\operatorname{det} \mathbf{M}_{g}^{\alpha}>1$ represents a net mass deposition and $\operatorname{det} \mathbf{M}_{g}^{\alpha}<1$ represents a net mass resorption. Using (14), the continuity equation $(10)_{1}$ may be written in local form as

$$
\rho^{\alpha} J_{*}^{\alpha}=\rho_{0}^{\alpha}
$$

where $\mathbf{J}_{*}^{\alpha}=\operatorname{det} \mathbf{F}_{l}^{\alpha} \operatorname{det} \mathbf{M}_{e}^{\alpha}$. In other words, the density change of $C^{\alpha}$ depends only on the effective elastic deformation. The balance of mass, linear momentum, and angular momentum for the mixture require that

$$
\sum_{\alpha=1}^{v-1} \rho^{\alpha} c^{\alpha}=\rho c, \quad \sum_{\alpha=1}^{v} \pi^{\alpha}=\mathbf{0}, \quad \sum_{\alpha=1}^{v} \Lambda^{\alpha}=\mathbf{0}
$$


where $\mathrm{c}$ is the mass growth function for the mixture. The balance of energy equation for the mixture is

$$
\sum_{\alpha=1}^{v}\left[\rho^{\alpha} \frac{\mathrm{d}^{\alpha} \varepsilon^{\alpha}}{\mathrm{dt}}-\rho^{\alpha} \mathbf{r}^{\alpha}+\operatorname{div} \mathbf{q}^{\alpha}+\pi^{\alpha} \cdot \mathbf{v}^{\alpha}-\mathbf{T}^{\alpha} \cdot \mathbf{L}^{\alpha}\right]-\sum_{\alpha=1}^{v-1} \rho^{\alpha} \beta^{\alpha}=0 .
$$

In the present paper, the constitutive theory that we use is based on the approach of Krishnaswamy and Batra (1997) for a solid-fluid mixture at a common temperature. In that approach, a special process was defined (called a homothermal quasi-static process) which was assumed to be reversible, and used with the Second Law of Thermodynamics to obtain a prescription for the partial entropy function.

In Klisch and Hoger (2000b), that approach was used to develop a theory for a mixture of $v-1$ elastic materials and an inviscid fluid at a common temperature. In particular, for a mixture $\mathrm{m}$ we define

$$
\Delta=\left\{\mathbf{F}^{1}, \ldots, \mathbf{F}^{v^{-1}}, \rho^{v}, \mathbf{G}^{1}, \ldots, \mathbf{G}^{v^{-1}}, \operatorname{grad} \rho^{v}, \theta\right\}
$$

where $\mathbf{G}^{\alpha}=\operatorname{Grad} \mathbf{F}^{\alpha}$. Furthermore, for a mixture $\mathrm{m}$,

$$
\begin{aligned}
& \mathbf{T}^{\alpha}=\mathrm{o}^{\alpha}(\Delta)+\mathrm{e} \mathbf{T}^{\alpha}\left(\Delta, \mathbf{a}^{1}, \ldots, \mathbf{a}^{v-1}\right), \quad \mathrm{e} \mathbf{T}^{\alpha}(\Delta, \mathbf{0}, \ldots, \quad \mathbf{0})=\mathbf{0}, \\
& \boldsymbol{\pi}^{\alpha}=\mathrm{o}_{\mathrm{o}}^{\alpha}(\Delta)+\mathrm{e} \boldsymbol{\pi}^{\alpha}\left(\Delta, \mathbf{a}^{1}, \ldots, \mathbf{a}^{v-1}\right), \quad \mathrm{e} \boldsymbol{\pi}^{\alpha}(\Delta, \mathbf{0}, \ldots, \mathbf{0})=\mathbf{0}, \\
& \psi^{\alpha}=\psi^{\alpha}(\Delta)=\varepsilon^{\alpha}-\eta^{\alpha} \theta,
\end{aligned}
$$

where $\psi^{\alpha}$ is the partial Helmholtz free energy function and $\eta^{\alpha}$ is the partial entropy.

The theory for a mixture $\mathrm{m}$ was then extended to develop a growth mixture theory. Following the development of a theory of growth for thermoelastic materials presented in Klisch and Hoger (2000a), we did not assume that reversible processes exist for a growing mixture. Motivated by the assumption that the material deposited for $C^{\alpha}$ during the growth process has the same mechanical properties as the original material of $C^{\alpha}$, we assumed that a growing mixture $\mathrm{m}_{\mathrm{g}}$ inherits its response functions for partial free energy, partial stress, diffusive force, partial entropy, and partial heat flux from those of a unique mixture $\mathrm{m}$, with an exception: the dependence on $\mathbf{F}^{\alpha}$ and $\mathbf{G}^{\alpha}$ in the response functions for $\mathrm{m}$ is replaced by a dependence on the effective elastic deformation $\mathbf{F}_{*}^{\alpha}$ and its gradient $\mathbf{G}_{*}^{\alpha}$ for $\mathrm{m}_{\mathrm{g}}$. Thus, for $\mathrm{m}_{\mathrm{g}}$ we define

$$
\Delta *=\left\{\mathbf{F}_{*}^{1}, \ldots, \mathbf{F}_{*}^{v-1}, \mathbf{G}_{*}^{1}, \ldots, \mathbf{G}_{*}^{v-1}, \rho^{v}, \operatorname{grad} \rho^{v}, \theta\right\}
$$

Consequently, each growing mixture $\mathrm{m}_{\mathrm{g}}$ is associated with a mixture $\mathrm{m}$ such that

$$
\begin{gathered}
\psi_{\mathrm{mg}}^{\alpha}(\Delta *)=\psi_{\mathrm{m}}^{\alpha}(\Delta), \quad{ }_{\mathrm{o}} \mathbf{T}_{\mathrm{mg}}^{\alpha}(\Delta *)={ }_{\mathrm{o}} \mathbf{T}_{\mathrm{m}}^{\alpha}(\Delta), \quad \mathrm{e} \mathbf{T}_{\mathrm{mg}}^{\alpha}\left(\Delta *, \mathbf{a}^{1}, \ldots, \mathbf{a}^{v-1}\right)=\mathrm{e} \mathbf{T}_{\mathrm{m}}^{\alpha}\left(\Delta, \mathbf{a}^{1}, \ldots, \mathbf{a}^{v-1}\right), \\
{ }_{\mathrm{o}} \boldsymbol{\pi}_{\mathrm{mg}}^{\alpha}(\Delta *)={ }_{\mathrm{o}} \boldsymbol{\pi}_{\mathrm{m}}^{\alpha}(\Delta), \quad \text { e } \pi_{\mathrm{mg}}^{\alpha}\left(\Delta *, \mathbf{a}^{1}, \ldots, \mathbf{a}^{v-1}\right)=\mathrm{e} \boldsymbol{\pi}_{\mathrm{m}}^{\alpha}\left(\Delta, \mathbf{a}^{1}, \ldots, \mathbf{a}^{v-1}\right), \\
\eta_{\mathrm{mg}}^{\alpha}(\Delta *)=\eta_{\mathrm{m}}^{\alpha}(\Delta), \quad \mathbf{q}_{\mathrm{mg}}^{\alpha}(\Delta *, \operatorname{grad} \theta)=\mathbf{q}_{\mathrm{m}}^{\alpha}(\Delta, \operatorname{grad} \theta) .
\end{gathered}
$$

When applied to (24), the constitutive restrictions derived, from thermodynamic considerations, for a mixture yield (Klisch and Hoger, 2000b)

$$
\eta_{m_{g}}^{\alpha}=-\frac{\partial \psi_{m_{g}}^{\alpha}}{\partial \theta}
$$




$$
\begin{gathered}
{ }_{\mathrm{o}} \mathbf{T}_{\mathrm{m}_{\mathrm{g}}}^{\alpha}=\sum_{\beta=1}^{v} \rho^{\beta} \frac{\partial \psi_{\mathrm{mg}_{\mathrm{g}}}^{\beta}}{\partial \mathbf{F}_{*}^{\alpha}} \mathbf{F}_{*}^{\alpha^{\mathrm{T}}} \quad(\alpha=1, \ldots, v-1), \quad{ }_{\mathrm{o}} \mathbf{T}_{\mathrm{m}_{\mathrm{g}}}^{v}=-\sum_{\beta=1}^{v} \rho^{\beta} \rho^{v} \frac{\partial \psi_{\mathrm{mg}_{\mathrm{g}}}^{\beta}}{\partial \rho^{v}} \mathbf{1}, \\
{ }_{\mathrm{o}} \boldsymbol{\pi}_{\mathrm{mg}}^{\alpha}=-\sum_{\beta=1}^{v-1}\left(\rho^{\alpha} \frac{\partial \psi_{\mathrm{mg}}^{\alpha}}{\partial \mathbf{F}_{*}^{\beta}} \operatorname{grad} \mathbf{F}_{*}^{\beta}-\rho^{\beta} \frac{\partial \psi_{\mathrm{mg}}^{\beta}}{\partial \mathbf{F}_{*}^{\alpha}} \operatorname{grad} \mathbf{F}_{*}^{\alpha}\right)-\rho^{\alpha} \frac{\partial \psi_{\mathrm{mg}}^{\alpha}}{\partial \rho^{v}} \operatorname{grad} \rho^{v}+\rho^{v} \frac{\partial \psi_{\mathrm{mg}}^{v}}{\partial \mathbf{F}_{*}^{\alpha}} \operatorname{grad} \mathbf{F}_{*}^{\alpha} .
\end{gathered}
$$

Following the theory for a growing elastic material (Klisch et al., 2000) we assume that the material time-derivative $\mathrm{d}^{\alpha}(\cdot) / \mathrm{dt}$ of $\mathbf{M}_{g}^{\alpha}$ is independent of the rates associated with the superposed elastic deformations $\mathbf{F}_{l}^{\beta}$. Thus, we assume a constitutive equation of the form

$$
\frac{\mathrm{d}^{\alpha} \mathbf{M}_{g}^{\alpha}}{\mathrm{dt}}=\hat{\mathbf{G}}^{\alpha}\left(\mathbf{T}^{\beta}, \mathbf{F}_{l}^{\beta}, \mathbf{M}_{e}^{\beta}, \mathbf{M}_{g}^{\beta}, \mathbf{a}^{\beta}, \theta, \operatorname{grad} \theta\right) .
$$

Lastly, we assume that the growth energy supply depends on the same variables as in (28): ${ }^{4,5}$

$$
\beta^{\alpha}=\tilde{\beta}^{\alpha}\left(\mathbf{T}^{\beta}, \mathbf{F}_{l}^{\beta}, \mathbf{M}_{e}^{\beta}, \mathbf{M}_{g}^{\beta}, \mathbf{a}^{\beta}, \theta, \operatorname{grad} \theta\right) .
$$

Finally, in (Klisch and Hoger, 2000b) we considered two special types of mechanical constraints that involve the deformations of the constituents. We generalized a procedure first introduced by Adkins (1958) and further developed by Truesdell and Noll (1965) for introducing internal constraints in finite elasticity ${ }^{6}$. For a growing mixture subject to a mechanical constraint, we assume that the partial stresses and diffusive forces obey the additive decompositions

$$
\mathbf{T}^{\alpha}=\overline{\mathbf{T}}^{\alpha}+\hat{\mathbf{T}}^{\alpha}, \quad \pi^{\alpha}=\overline{\boldsymbol{\pi}}^{\alpha}+\hat{\pi}^{\alpha},
$$

where $\left(\overline{\mathbf{T}}^{\alpha}, \overline{\boldsymbol{\pi}}^{\alpha}\right)$ are indeterminate partial stresses and diffusive forces called the constraint responses, and $\left(\hat{\mathbf{T}}^{\alpha}, \hat{\boldsymbol{\pi}}^{\alpha}\right)$ are determinate partial stresses and diffusive forces. Furthermore, we assume that the work done by the constraint response functions is zero, i.e.,

$$
\sum_{\alpha=1}^{v}\left(\overline{\mathbf{T}}^{\alpha} \cdot \mathbf{L}^{\alpha}-\bar{\pi}^{\alpha} \cdot \mathbf{a}^{\alpha}\right)=0
$$

Two special types of internal constraints will be included in the cartilage growth model presented in the next section. For simplicity, we restrict the discussion to a mixture of two growing elastic materials and an inviscid fluid.

\section{Example 1: Equal total deformation gradients for the growing elastic materials}

The requirement that the overall deformation gradient tensors of two growing elastic materials $(\alpha=1,2)$ be identical results in the nine constraint equations

$$
\phi_{\mathrm{ij}}=\mathbf{e}_{\mathrm{i}} \cdot \mathbf{F}^{1} \mathbf{e}_{\mathrm{j}}-\mathbf{e}_{\mathrm{i}} \cdot \mathbf{F}^{2} \mathbf{e}_{\mathrm{j}}=0, \quad(\mathrm{i}, \mathrm{j}=1,2,3),
$$

\footnotetext{
${ }^{4}$ In Klisch and Hoger (2000b), an equivalence relation on the set of all growing mixtures was defined such that a mixture $\mathrm{m}$ generates an equivalence class, or family, of growing mixtures $\mathrm{m}_{\mathrm{g}}$ which differ through their growth response functions (28) and (29).

${ }^{5}$ Additional constitutive restrictions imposed by the Second Law of Thermodynamics were derived in Klisch and Hoger (2000b), but are not needed here.

${ }^{6}$ Adkins (1958) assumed that the indeterminate response for the stress was symmetric, whereas Truesdell and Noll (1965). provided a geometric argument to obtain the constraint response and proved that the indeterminate stress must be symmetric for a finitely elastic material.
} 
where $\left(\mathbf{e}_{1}, \mathbf{e}_{2}, \mathbf{e}_{3}\right)$ is a fixed orthonormal basis. The material time derivative of the nine equations (32) with respect to $C^{1}$ are each introduced with a Lagrange multiplier into the condition (31). Noting that, due to the constraint, $\mathbf{L}^{1}=\mathbf{L}^{2}$ and $\mathbf{a}^{1}=\mathbf{a}^{2}$, so we obtain

$$
\overline{\mathbf{T}}^{1}=-\overline{\mathbf{T}}^{2}=\bar{\lambda}, \quad \overline{\boldsymbol{\pi}}^{1}=-\overline{\boldsymbol{\pi}}^{2}=\overline{\mathbf{p}},
$$

where $\bar{\lambda}$ and $\overline{\mathbf{p}}$ are arbitrary.

\section{Example 2: Intrinsic Incompressibility}

A commonly used constraint in the theory of mixtures is that of intrinsic incompressibility, which was first proposed by Mills (1966). Each constituent $C^{\alpha}$ is assumed to be separable from the others with constant (true) density $\rho^{\alpha^{\mathrm{T}}}$ defined as the mass of $C^{\alpha}$ per unit volume of $C^{\alpha}$. It is assumed that the volume of the mixture is equal to the sum of the volumes of each $C^{\alpha}$. With these assumptions, Mills (1966) derived the constraint equation:

$$
\phi=\sum_{\alpha=1}^{3}\left\{\frac{\rho^{\alpha}}{\rho^{\alpha \mathrm{T}}}\right\}-1=0 .
$$

The material time derivative of (34) with respect to the fluid constituent $(\alpha=3)$ is introduced with a Lagrange multiplier $\mathrm{p}$ into the condition (31). Noting that $\mathbf{L}_{l}^{\alpha}$ may be chosen independently from $\mathbf{L}_{l}^{\beta}(\beta \neq \alpha)$

and $\left(\mathbf{L}_{e}^{1}, \mathbf{L}_{g}^{1}, \mathbf{a}^{1}, \mathbf{L}_{e}^{2}, \mathbf{L}_{g}^{2}, \mathbf{a}^{2}, \mathbf{L}^{3}\right)$, it follows that

$$
\overline{\mathbf{T}}^{\alpha}=\mathrm{p} \frac{\rho^{\alpha}}{\rho^{\alpha \mathrm{T}}} \mathbf{1}, \quad \sum_{\alpha=1}^{2}\left\{\mathrm{p} \frac{\rho^{\alpha}}{\rho^{\alpha \mathrm{T}}} \mathrm{c}^{\alpha}-\left(\overline{\boldsymbol{\pi}}^{\alpha}+\mathrm{p} \frac{\operatorname{grad} \rho^{\alpha}}{\rho^{\alpha \mathrm{T}}}\right) \cdot \mathbf{a}^{\alpha}\right\}=0 .
$$

Recalling the growth continuity equation (15), it is apparent that we may derive a response function for $\mathrm{c}^{\alpha}$ from the material time-derivative $\mathrm{d}^{\alpha}(\cdot) / \mathrm{dt}$ of $\mathbf{M}_{g}^{\alpha}$ (28). As this latter response function is allowed to depend on the relative velocities $\mathbf{a}^{\alpha}$, the derived response function for $\mathrm{c}^{\alpha}$ may also depend on $\mathbf{a}^{\alpha}$. Thus, for the general case no further reduction is possible for $(35)_{2}$. However, it seems plausible to suggest that $\mathrm{c}^{\alpha}$ only depends on the magnitude of the relative velocities. Thus, consider the following special assumption concerning the derived response function for each $c^{\alpha}: c^{\alpha}\left(\mathbf{a}^{1}\right)=c^{\alpha}\left(-\mathbf{a}^{1}\right)$ and $c^{\alpha}\left(\mathbf{a}^{2}\right)=c^{\alpha}\left(-\mathbf{a}^{2}\right)$. For this special assumption, $(35)_{2}$ reduces to

$$
\bar{\pi}^{\alpha}=-p \frac{\operatorname{grad} \rho^{\alpha}}{\rho^{\alpha T}} .
$$

In the cartilage growth model, we assume that (36) holds.

\section{THE CARTILAGE GROWTH MODEL}

In this section, we present the reduced constitutive equations that are used to study the growth of cartilaginous tissues in the example presented in the next section. The model contains a solid matrix composed of two elastic materials, representing the collagen and the proteoglycan constituents, and an inviscid fluid that represents the water and dissolved solutes. This particular model is motivated by the observation that the collagen and proteoglycan constituents may grow independently of each other, while experiencing the same overall deformation. Figure 2 shows an idealization of this model for the example problem presented in the next section, where a sphere of cartilage grows isotropically into a larger sphere of cartilage in the absence of external loading. The collagen and proteoglycan constituents experience distinct growth tensors $\mathbf{M}_{g}^{\mathrm{C}}$ and $\mathbf{M}_{g}^{\mathrm{P}}$, respectively, during which the density of each constituent remains unchanged. 
When the amounts of growth for these constituents are different, the constituents must experience distinct elastic deformations $\mathbf{M}_{e}^{\mathrm{C}}$ and $\mathbf{M}_{e}^{\mathrm{P}}$, as illustrated in Figure 2. These elastic deformations must satisfy the constraint that the overall deformations of the constituents are equal $\left(\mathbf{F}^{\mathrm{P}}=\mathbf{F}^{\mathrm{C}}\right)$, and the governing equations for the corresponding boundary-value problem.

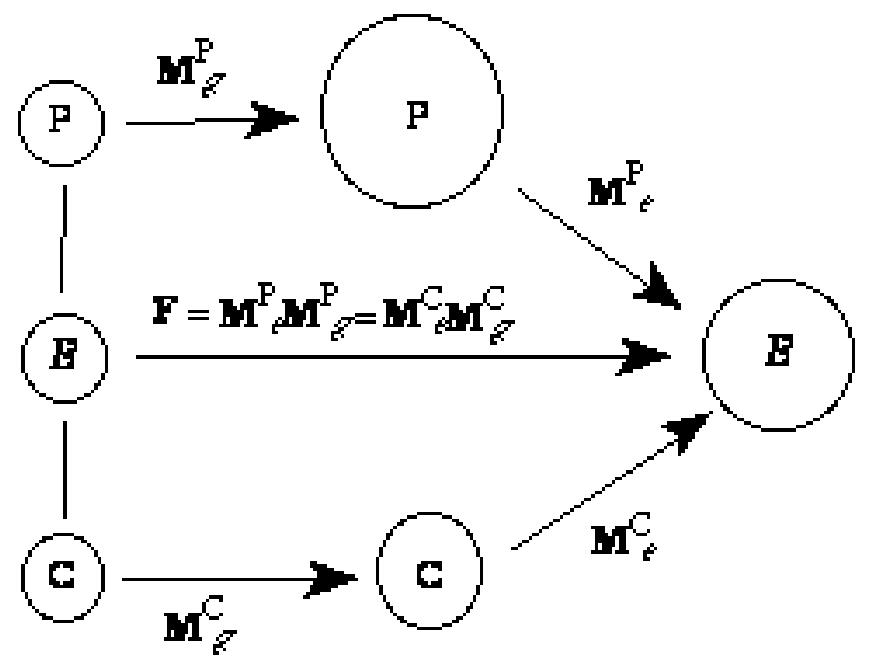

Figure 2: Idealized cartilage growth model.

The cartilage growth model is formally derived by applying the following conditions to the theory presented in the previous sections:

(i) The mixture contains three materials, with superscripts P, C, and F denoting the proteoglycan, collagen, and fluid constituents. The $\mathrm{P}$ and $\mathrm{C}$ constituents are growing elastic materials, while the F constituent is an inviscid fluid.

(ii) We adopt the internal constraint that the overall deformation gradient tensors of the $\mathrm{P}$ and $\mathrm{C}$ constituents is identical, i.e., $\mathbf{F}^{\mathrm{P}}=\mathbf{F}^{\mathrm{C}}$. Consequently, the relative velocities $\mathbf{a}^{\mathrm{P}}$ and $\mathbf{a}^{\mathrm{C}}$ are equal; we set $\mathbf{a}=\mathbf{a}^{\mathrm{P}}=\mathbf{a}^{\mathrm{C}}$.

(iii) We adopt the internal constraint of intrinsic incompressibility.

(iv) The gradients of the effective elastic tensors and the fluid density $\left(\mathbf{G}_{*}^{\mathrm{P}}, \mathbf{G}_{*}^{\mathrm{C}}, \operatorname{grad} \rho^{\mathrm{F}}\right)$ are not included among the list of independent variables (23). Furthermore, the partial stresses and the diffusive forces are linear functions of the relative velocities. Thus, we define

$$
\Delta *=\left\{\mathbf{F}_{*}^{\mathrm{P}}, \mathbf{F}_{*}^{\mathrm{C}}, \rho^{\mathrm{F}}, \theta\right\}, \quad \mathbf{T}^{\alpha}=\mathrm{o} \mathbf{T}^{\alpha}(\Delta *)+\mathbf{T}^{\alpha}(\Delta *) \mathbf{a}, \quad \pi^{\alpha}=0 \pi^{\alpha}(\Delta *)+\mathrm{e} \pi^{\alpha}(\Delta *) \mathbf{a}
$$

Condition (iv) follows an approach advocated by Atkin and Craine (1976) for simplifying the constitutive theory in an attempt to more easily solve boundary-value problems. This represents a limitation of the present model, a point that is discussed further in the concluding remarks of this paper.

Introducing the conditions (i-iv) into the constitutive theory presented above specialized to a mixture of two elastic materials and an inviscid fluid, we obtain the results

$$
\frac{\partial \psi^{\mathrm{P}}}{\partial \rho^{\mathrm{F}}}=\frac{\partial \psi^{\mathrm{C}}}{\partial \rho^{\mathrm{F}}}=0, \quad \frac{\partial \psi^{\mathrm{P}}}{\partial \mathbf{F}_{*}^{\mathrm{C}}}=\frac{\partial \psi^{\mathrm{C}}}{\partial \mathbf{F}_{*}^{\mathrm{P}}}=\frac{\partial \psi^{\mathrm{F}}}{\partial \mathbf{F}_{*}^{\mathrm{P}}}=\frac{\partial \psi^{\mathrm{F}}}{\partial \mathbf{F}_{*}^{\mathrm{C}}}=\mathbf{0},
$$

and 


$$
\mathrm{e}^{\alpha}(\Delta *)=\mathbf{0} \Rightarrow \mathbf{T}^{\alpha}=\mathrm{o}^{\alpha}(\Delta *)
$$

With (38-39) and the constraint response functions (33), (35), and (36), we obtain the entropy equation (25) and the reduced constitutive restrictions

$$
\begin{gathered}
\psi^{\alpha}=\psi^{\alpha}\left(\mathbf{F}_{*}^{\alpha}, \theta\right) \quad(\alpha=\mathrm{P}, \mathrm{C}), \quad \psi^{\mathrm{F}}=\psi^{\mathrm{F}}\left(\rho^{\mathrm{F}}, \theta\right), \\
\mathbf{T}^{\mathrm{P}}=\mathrm{p} \frac{\rho^{\mathrm{P}}}{\rho^{\mathrm{PT}}} \mathbf{1}+\bar{\lambda}+\rho^{\mathrm{P}} \frac{\partial \psi^{\mathrm{P}}}{\partial \mathbf{F}_{*}^{\mathrm{P}}} \mathbf{F}_{*}^{\mathrm{P}^{\mathrm{T}}}, \quad \mathbf{T}^{\mathrm{C}}=\mathrm{p} \frac{\rho^{\mathrm{C}}}{\rho^{\mathrm{CT}}} \mathbf{1}-\bar{\lambda}+\rho^{\mathrm{C}} \frac{\partial \psi^{\mathrm{C}}}{\partial \mathbf{F}_{*}^{\mathrm{C}}} \mathbf{F}_{*}^{\mathrm{C}^{\mathrm{T}}}, \quad \mathbf{T}^{\mathrm{F}}=\mathrm{p} \frac{\rho^{\mathrm{F}}}{\rho^{\mathrm{FT}}} \mathbf{1}-\left(\rho^{\mathrm{F}}\right)^{2} \frac{\partial \psi^{\mathrm{F}}}{\partial \rho^{\mathrm{F}}} \mathbf{1}, \\
\pi^{\mathrm{P}}=-\mathrm{p} \frac{\operatorname{grad} \rho^{\mathrm{P}}}{\rho^{\mathrm{PT}}}+\overline{\boldsymbol{\pi}}+\mathrm{e} \boldsymbol{\pi}^{\mathrm{P}} \mathbf{a}, \quad \boldsymbol{\pi}^{\mathrm{C}}=-\mathrm{p} \frac{\operatorname{grad} \rho^{\mathrm{C}}}{\rho^{\mathrm{CT}}}-\overline{\boldsymbol{\pi}}+\mathrm{e} \boldsymbol{\pi}^{\mathrm{C}} \mathbf{a}, \quad \boldsymbol{\pi}^{\mathrm{F}}=-\boldsymbol{\pi}^{\mathrm{P}}-\pi^{\mathrm{C}} .
\end{gathered}
$$

Furthermore, an entropy inequality may be used to produce additional restrictions.

Thus, the cartilage growth model contains two elastic constituents that may grow independently of each other in response to mechanical stimuli including stress, strain, and fluid flow. As these constituents grow, they may develop distinct elastic deformations that alter their stresses, their relative concentrations, and the overall mechanical response of the tissue.

\section{EXAMPLE}

In this section, we present an example that illustrates several of the main features of the cartilage growth model proposed above. Although external loading may serve to drive the growth process in biological tissues, in this example we restrict ourselves to studying the evolution of the material in an unloaded configuration and take the growth tensors to be independent of mechanical quantities. Thus, we specify the growth tensors for each constituent, as opposed to determining them from their growth response functions.

\section{Boundary-value problem}

We consider the uniform and isotropic growth of a sphere of cartilage for which the initial and final configurations are in equilibrium in the absence of external loads and body forces. For the purpose of this example, we select a specific form for the growth tensors $\mathbf{M}_{g}^{\mathrm{C}}$ and $\mathbf{M}_{g}^{\mathrm{P}}$ and determine the effective elastic deformations $\mathbf{M}_{e}^{\mathrm{C}}$ and $\mathbf{M}_{e}^{\mathrm{P}}$ that satisfy the equilibrium equations and the constraint that the overall deformation gradient tensors are identical. For a realistic growth problem, the forms of $\mathbf{M}_{g}^{\mathrm{C}}$ and $\mathbf{M}_{g}^{\mathrm{P}}$ would be obtained from the growth response functions; the rest of the boundary-value problem would be solved as we do here.

Thus, we specify the isotropic growth tensors,

$$
\mathbf{M}_{g}^{\mathrm{P}}=\mathrm{g}^{\mathrm{P}} \mathbf{1}, \quad \mathbf{M}_{g}^{\mathrm{C}}=\mathrm{g}^{\mathrm{C}} \mathbf{1},
$$

where $\mathrm{g}^{\mathrm{P}}$ and $\mathrm{g}^{\mathrm{C}}$ are the growth stretches. We emphasize that the growth stretches are assumed to be homogeneous and may be specified independently of each other.

Since we seek equilibrium solutions, the relative velocity vanishes, i.e., $\mathbf{a}=\mathbf{0}$. Also, we assume that the indeterminate diffusive force $\bar{\pi}$ and the indeterminate multiplier $\mathrm{p}$ due to the intrinsic incompressibility constraint vanish, so that the diffusive forces (42) are zero at equilibrium. There are no superposed elastic deformation tensors due to loading, so $\mathbf{F}_{l}^{\mathrm{P}}=\mathbf{F}_{l}^{\mathrm{C}}=\mathbf{1}$.

Let the constituent volume fractions be defined by

$$
\phi^{\alpha}=\frac{\rho^{\alpha}}{\rho^{\alpha \mathrm{T}}} .
$$

Then we can rewrite the balance of mass and intrinsic incompressibility constraint equations in terms of $\phi^{\alpha}$. Thus, we must satisfy the following balance of mass and equilibrium equations: 


$$
\phi^{\mathrm{P}} \mathrm{J}_{\mathrm{e}}^{\mathrm{P}}=\phi_{0}^{\mathrm{P}}, \quad \phi^{\mathrm{C}} \mathrm{J}_{\mathrm{e}}^{\mathrm{C}}=\phi_{0}^{\mathrm{C}}, \quad \operatorname{div} \mathbf{T}^{\mathrm{P}}=0, \quad \operatorname{div} \mathbf{T}^{\mathrm{C}}=0,
$$

where $\mathbf{J}_{e}^{\alpha}=\operatorname{det} \mathbf{M}_{e}^{\alpha}$. Once (45), are satisfied, the fluid volume fraction $\phi^{\mathrm{F}}$ may be calculated from the intrinsic incompressibility constraint (34). In this example, the tensors $\mathbf{M}_{e}^{\alpha}$ represent the elastic deformations required to maintain the constraints (32). We look for homogeneous, isotropic solutions of the form

$$
\mathbf{M}_{e}^{\mathrm{P}}=\lambda^{\mathrm{P}} \mathbf{1}, \quad \mathbf{M}_{e}^{\mathrm{C}}=\lambda^{\mathrm{C}} \mathbf{1},
$$

where $\lambda^{\mathrm{P}}$ and $\lambda^{\mathrm{C}}$ are the elastic stretches. In order to satisfy the constraints (32), the collagen elastic stretch must satisfy

$$
\lambda^{\mathrm{C}}=\lambda^{\mathrm{P}}\left(\mathrm{g}^{\mathrm{P}} / \mathrm{g}^{\mathrm{C}}\right) \equiv \lambda^{\mathrm{P}} \mathrm{g}^{\mathrm{P} / \mathrm{C}}
$$

where $\mathrm{g}^{\mathrm{P} / \mathrm{C}}$ is called the growth ratio. The boundary condition is that the total traction must vanish on the boundary. Due to the assumptions of homogeneous and isotropic elastic stretches, this requires the total stress to vanish everywhere:

$$
\mathbf{T}^{\mathrm{P}}+\mathbf{T}^{\mathrm{C}}+\mathbf{T}^{\mathrm{F}}=\mathbf{0} .
$$

\section{Constitutive equations}

We look for solutions such that the indeterminate stress terms vanish; i.e., $\bar{\lambda}=\mathbf{0}$ and, as stated above, $\mathrm{p}=0$. A constant fluid free energy function is assumed (see Klisch and Lotz (2000) for a discussion regarding this assumption); thus, $(41)_{3}$ and $\mathrm{p}=0$ results in $\mathbf{T}^{\mathrm{F}}=\mathbf{0}$ at every point. The proteoglycan stress constitutive equation is derived using the theoretical analysis of Basser et al. (1998) and data obtained in our laboratory from adult bovine articular cartilage harvested from the condyle of the knee (Williamson et al., 2000). The tissue's aggregate modulus $\mathrm{H}_{\mathrm{A} 0}$ is $0.31 \mathrm{MPa}$ (i.e., $\mathrm{H}_{\mathrm{A} 0}$ equals the initial slope of the tissue stress-stretch equation for a confined compression protocol) and average initial contents are $\left(\phi_{0}^{\mathrm{F}}, \phi_{0}^{\mathrm{P}}, \phi_{0}^{\mathrm{C}}\right)=$ $(0.83,0.02,0.15)$. Assuming that the proteoglycan stress only depends on the determinant of its elastic deformation, we obtain

$$
\mathbf{T}^{\mathrm{P}}=\frac{\gamma_{1}}{\left(\mathrm{~J}_{e}^{\mathrm{P}}\right)^{\gamma_{2}}} \mathbf{1}
$$

with $\gamma_{1}=-0.071 \mathrm{MPa}$ and $\gamma_{2}=2.108$. The collagen stress constitutive equation is formed by noting that the solid matrix stress is equal to the sum of the proteoglycan and collagen stresses. The solid matrix stress is derived from an isotropic strain energy function obtained from Almeida and Spilker (1998):

$$
\mathrm{W}=\frac{\alpha_{0}}{\left(\mathrm{I}_{3}\right)^{\beta}} \exp \left[\alpha_{1}\left(\mathrm{I}_{1}-3\right)+\alpha_{2}\left(\mathrm{I}_{2}-3\right)+\alpha_{3}\left(\mathrm{I}_{1}-3\right)^{2}\right]
$$

where $I_{i}(i=1,2,3)$ are the principal invariants of the Cauchy-Green tensor and $\left(\alpha_{0}, \alpha_{1}, \alpha_{2}, \alpha_{3}, \beta\right)$ are material constants equal to $(0.010,7.733,-3.562,3.578,0.609)$ (the material constants were selected to provide $\mathrm{H}_{\mathrm{A} 0}=$ $0.31 \mathrm{MPa}$ and to satisfy certain constitutive inequalities). Thus, we postulate the collagen stress equation

$$
\mathbf{T}^{\mathrm{C}}=\frac{2}{\left(\mathrm{I}_{3}^{\mathrm{C}}\right)^{1 / 2}}\left[\left(\mathrm{I}_{2}^{\mathrm{C}} \mathrm{W}_{2}+\mathrm{I}_{3}^{\mathrm{C}} \mathrm{W}_{3}\right) \mathbf{1}+\mathrm{W}_{1} \mathbf{B}_{e}^{\mathrm{C}}-\mathrm{I}_{3}^{\mathrm{C}} \mathrm{W}_{2}\left(\mathbf{B}_{e}^{\mathrm{C}}\right)^{-1}\right]-\frac{\gamma_{1}}{\left(\mathrm{~J}_{e}^{\mathrm{C}}\right)^{\gamma_{2}}} \mathbf{1},
$$


where $\mathbf{B}_{e}^{\mathrm{C}}$ is the collagen left Cauchy-Green tensor and $\mathrm{W}_{\mathrm{i}}$ is the partial derivative of $\mathrm{W}$ with respect to the ith invariant of $\mathbf{B}_{e}^{\mathrm{C}}$. Eqn. (51) is proposed so that when the elastic deformations of the proteoglycan and collagen constituents are equal (e.g., when no growth occurs), the sum of the stresses (49) and (51) yields that of the solid matrix stress derived from (50).

\section{Solution procedure}

To solve the growth boundary-value problem, we first specify $\mathrm{g}^{\mathrm{P} / \mathrm{C}}$. Using $(46)_{1}$, the proteoglycan stress (49) is written as a function of $\lambda^{\mathrm{P}}$. With $(46)_{2}$ and the constraint (47), the collagen stress (51) is written as a function of $\lambda^{\mathrm{P}}$ and $\mathrm{g}^{\mathrm{P} / \mathrm{C}}$. Noting that these stress tensors are necessarily spherical due to our assumptions, and recalling $\mathbf{T}^{\mathrm{F}}=\mathbf{0}$ and the boundary condition (48) we obtain an algebraic equation of the form

$$
f\left\{\lambda^{P}, g^{P / C}\right\}=0,
$$

which is solved numerically to determine the proteoglycan stretch $\lambda^{\mathrm{P}}$. Then, the collagen elastic stretch $\lambda^{\mathrm{C}}$ is calculated from (47) and the pre-stresses ${ }^{7}$ are calculated from (49) and (51). In addition, for each equilibrium state following a prescribed growth, the aggregate modulus $\mathrm{H}_{\mathrm{A} 0}$ of the solid matrix is calculated by superimposing identical confined compression deformations on the proteoglycan and collagen constituents, and evaluating the initial slope of the solid matrix stress vs. stretch curve in the direction of applied loading. Finally, the water content is calculated using the solutions for the elastic stretches, $(45)_{1,2}$, and the initial contents listed above.

\section{Results}

The calculated elastic stretches are shown in Figure 3 for various values of $g^{\mathrm{P} / \mathrm{C}}$. When $\mathrm{g}^{\mathrm{P} / \mathrm{C}}>1$, the proteoglycan experiences an isotropic compressive strain while the collagen experiences an isotropic tensile strain. The converse situation arises when $\mathrm{g}^{\mathrm{P} / \mathrm{C}}<1$; i.e., the proteoglycan experiences an isotropic tensile strain while the collagen experiences an isotropic compressive strain. These results are physically intuitive. A value of $\mathrm{g}^{\mathrm{P} / \mathrm{C}}>1$ corresponds to either of two scenarios: proteoglycan experiences more mass deposition than collagen, or collagen experiences more mass resorption than proteoglycan. Since isotropic uniform growth is a compatible deformation, an intermediate configuration for each constituent may be defined due to the growth alone. When more proteoglycan is deposited than collagen, the proteoglycan grows into a larger sphere than the collagen. Thus, to maintain the constraint (32) the proteoglycan should experience an elastic compression while the collagen should experience an elastic tension. This phenomenon is predicted by the solution.

The calculated Cauchy stress components are shown in Figure 4 for various values of $g^{\mathrm{P} / \mathrm{C}}$. Corresponding to the results obtained for the elastic stretches, when $\mathrm{g}^{\mathrm{P} / \mathrm{C}}>1$ the proteoglycan pre-stress becomes more compressive while the collagen pre-stress becomes more tensile. The converse situation arises when $\mathrm{g}^{\mathrm{P} / \mathrm{C}}<1$; i.e., the proteoglycan pre-stress becomes less compressive while the collagen pre-stress become less tensile.

The calculated solid matrix aggregate modulus $\mathrm{H}_{\mathrm{A} 0}$ is shown in Figure 5 for various values of $\mathrm{g}^{\mathrm{P} / \mathrm{C}}$. The aggregate modulus increases as $\mathrm{g}^{\mathrm{P} / \mathrm{C}}>1$ and, as $\mathrm{g}^{\mathrm{P} / \mathrm{C}}$ decreases, reaches a minimum value at approximately $\mathrm{g}^{\mathrm{P} / \mathrm{C}}=0.95$ and increases as $\mathrm{g}^{\mathrm{P} / \mathrm{C}}<0.95$. The calculated contents of the mixture are shown in Figure 6 for various values of $\mathrm{g}^{\mathrm{P} / \mathrm{C}}$.

\footnotetext{
${ }^{7}$ The term pre-stress refers to the constituent stress when there is no loading on the boundary of the mixture. Note that the constituent pre-stresses may be homogeneous, whereas a residual stress field for the solid matrix cannot be homogeneous. Thus, although the theory presented does not include residual stresses, it does allow for uniform constituent pre-stresses such that their sum must vanish.
} 


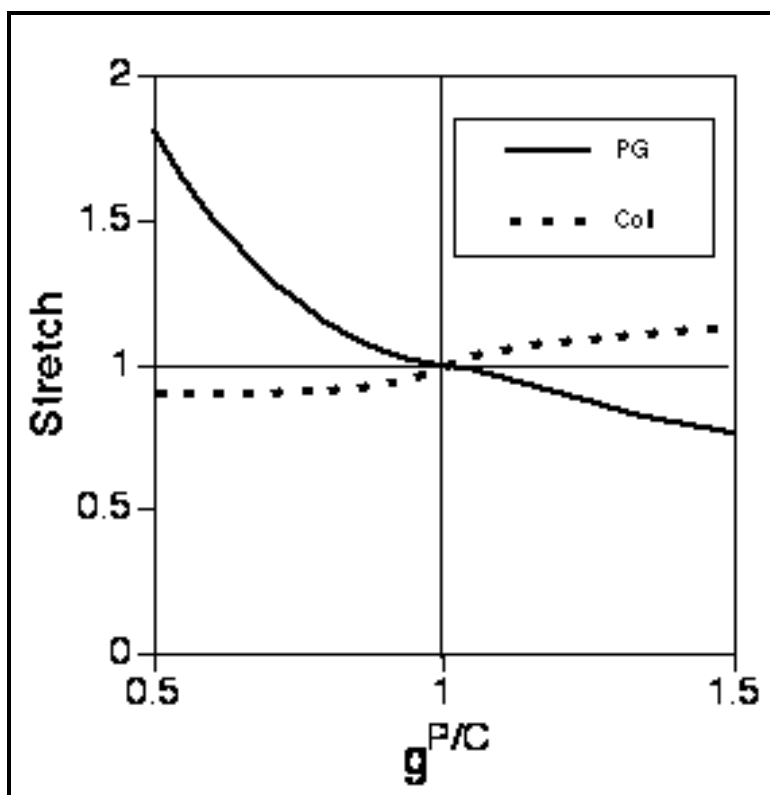

Figure 3: Elastic stretches vs. growth ratio.

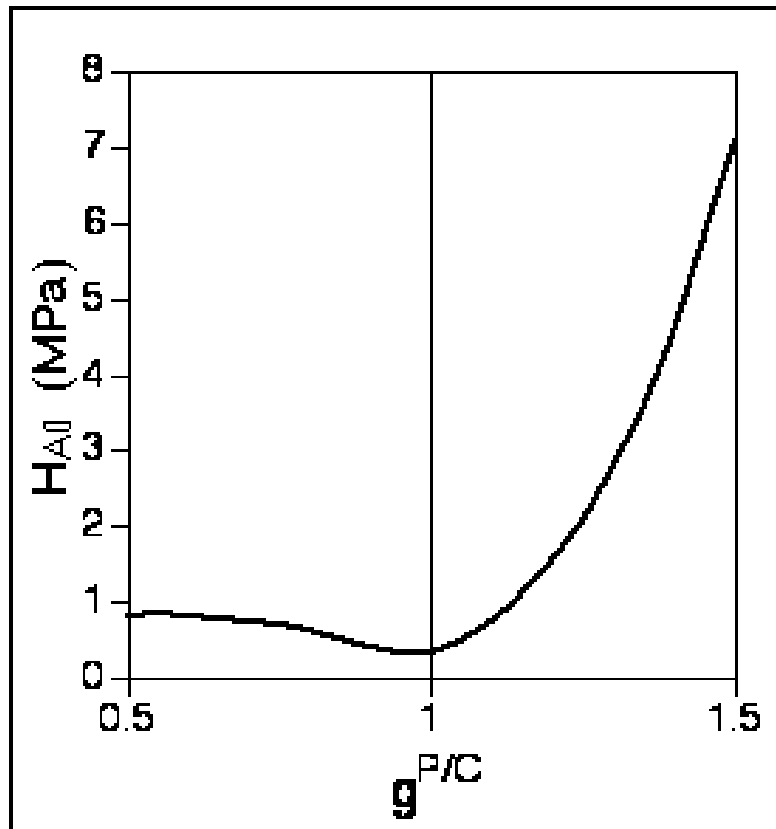

Figure 5: Aggregate modulus vs. growth ratio.

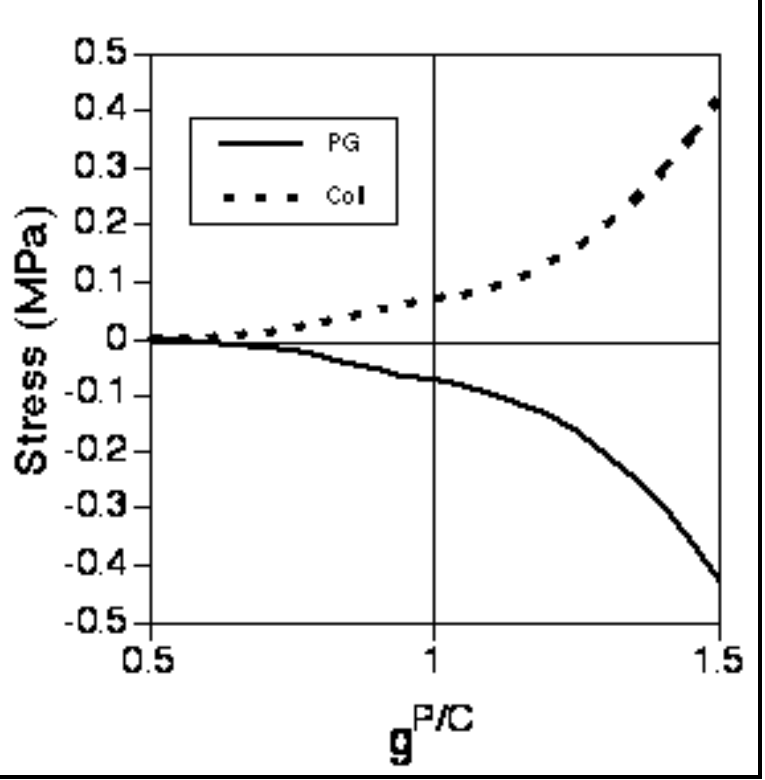

Figure 4: Pre-stresses vs. growth ratio.

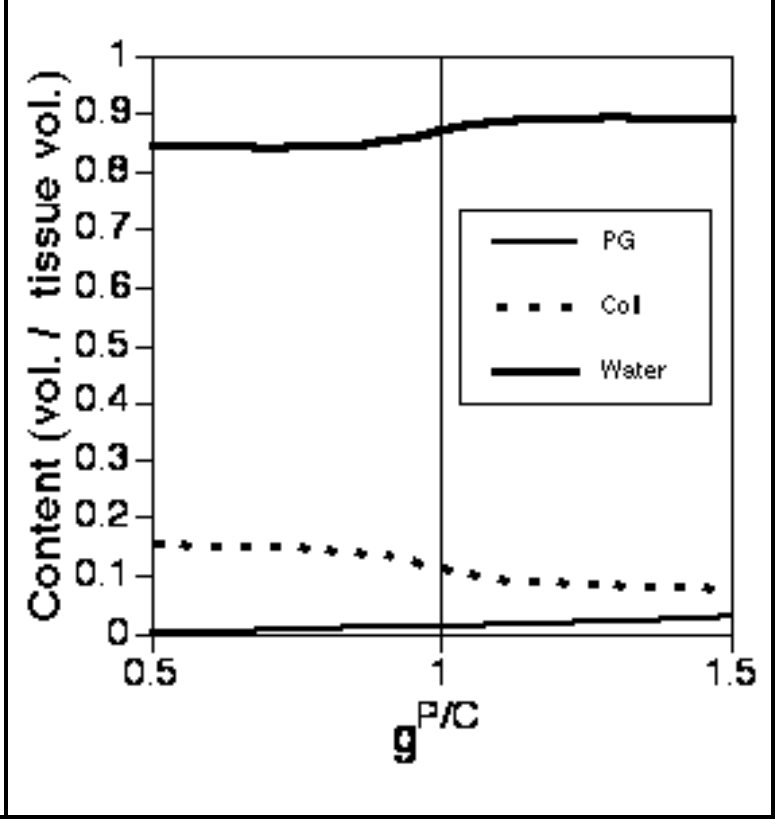

Figure 6: Contents vs. growth ratio. 


\section{CONCLUSION}

We have proposed a cartilage growth model based on a more general growth mixture theory and have illustrated the main features of the theory with an example problem. Two key elements of the cartilage growth model are that the proteoglycan and collagen constituents may grow independently of each other, and that their overall deformations must be identical. A limitation of the example is the uncertainty concerning the validity of the constitutive equations used for the proteoglycan and collagen stresses. Thus, future efforts should include the development of experimentally determined stress-strain equations. This aim may be achieved in parallel with an effort towards using the cartilage growth model to quantify the growth and remodeling of tissue-engineered cartilage. Another limitation of the present model is that we have neglected the gradients of the effective elastic tensors and the fluid density among the list of independent variables. This condition results in a proteoglycan stress equation that is independent of both the collagen elastic tensor and the fluid density. However, the theoretical model proposed by Basser et al. (1998) suggests that the proteoglycan stress depends on the densities of all three constituents. Finally, it has been shown that the cartilage microstructure changes during growth (Williamson et al., 2000), so it will be necessary to include internal variables in the cartilage growth model representing the collagen microstructure (e.g., fiber dimensions, fiber orientation, and crosslink density).

The example presented is restrictive and does not accurately reflect the actual growth process of cartilage in vivo or in vitro. In particular, the geometry in vivo is more complex than that presented here, and we have not studied the effects of anisotropic growth. Nevertheless, this special example illustrates several features of the cartilage growth model. First, the model allows the constituents in the mixture to grow independently of each other. Second, the model is capable of describing the evolution of the constituent pre-stresses due to growth. Third, the model may predict the evolution of the tissue composition (i.e., the relative concentrations of the constituents) and, consequently, the mechanical properties of the tissue during growth. The results indicate that the cartilage growth model is capable of predicting characteristics of the remodeling observed in numerous experiments which have established that changes in the tissue's mechanical properties and composition occur during both developmental growth and degeneration.

ACKNOWLEDGMENTS: We would like to thank Dr. Silvia Chen for assistance in developing the constitutive equations. The authors are grateful for support from the U.C. President's Office Postdoctoral Fellowship Program (SMK), the NIH (SMK and RLS), NASA (RLS), and the NSF (RLS and AH).

\section{REFERENCES}

Adkins, J.E., 1958, "Dynamic properties of resilient materials: constitutive equations," Philosophical Transactions of the Royal Society of London, Vol. 250A, pp. 519-541.

Almeida, E.S. and Spilker, R.L., 1998, "Finite element formulations for hyperelastic transversely isotropic biphasic soft tissues," Computer Methods and Applications in Mechanical Engineering, Vol. 151, pp. 513-538.

Ateshian, G.A., Warden, W.H., Kim, J.J., Grelsamer, R.P., and Mow, V.C., 1997, "Finite deformation biphasic material properties of bovine articular cartilage from confined compression experiments," Journal of Biomechanics, Vol. 30, pp. 1157-1164.

Atkin, R.J. and Craine, R.E., 1976, "Continuum theories of mixtures: Applications," Journal of the Institute of Mathematics and its Applications, Vol. 17, pp. 153-207.

Basser, P.J., Schneiderman, R., Bank, R.A., and Maroudas, A., 1998, "Mechanical properties of the collagen network in human articular cartilage as measured by osmotic stress technique," Archives of Biochemistry and Biophysics, Vol. 351, pp. 207-219.

Brickley-Parson, D. and Glimcher, J., 1984, "Is the chemistry of collagen in intervertebral discs an expression of Wolff's law?: a study of the human lumbar spine," Spine, Vol. 9, pp. 148-163.

Craine, R.E., Green, A.E., and Naghdi, P.M., 1970, "A mixture of viscous elastic materials with different constituent temperatures," Quarterly Journal of Mechanics and Applied Mathematics, Vol. 23, pp. 171-184.

Hoger, A., 2000, in review, "A theory of growth for soft biological tissues," Journal of Elasticity.

Kiviranta, I., Tammi, M., Jurvelin, J., Arokoski, J., Säämänen, A.M., and Helminen, H.J., 1994, “Articular cartilage thickness and glycosaminoglycan distribution in the young canine knee joint after remobilization of the immobilized limb," Journal of Orthopaedic Research, Vol. 12, pp. 161-167.

Klisch, S.M., Van Dyke, T., and Hoger, A., 2000, in review, "A theory of volumetric growth for compressible elastic biological materials," Mathematics and Mechanics of Solids.

Klisch, S.M. and Hoger, A., 2000a, in review, "A thermomechanical theory of growth, part I: Thermoelastic materials," Journal of Elasticity. 
Klisch, S.M. and Hoger, A., 2000b, in review, "A thermomechanical theory of growth, part II: A mixture of elastic materials and an inviscid fluid," Journal of Elasticity.

Klisch, S.M. and Lotz, J.C., 2000, "A special theory of biphasic mixtures and experimental results for human annulus fibrosus tested in confined compression," Journal of Biomechanical Engineering, Vol. 122, pp. 180-188.

Krishnaswamy, S. and Batra, R.C., 1997, "A thermomechanical theory of solid-fluid mixtures," Mathematics and Mechanics of Solids, Vol. 2, pp. 143-151.

Mills, N., 1966, "Incompressible mixtures of Newtonian fluids," International Journal of Engineering Science, Vol. 4, pp. 97-112.

Pauwels, F., 1976, "Biomechanics of the Normal and Diseased Hip," Springer-Verlag, New York.

Ogden, J.A., 1988, "Skeletal growth mechanism injury patterns," In: Behavior of the Growth Plate, Eds. Uhthoff, H.K. and Wiley, J.J., Raven Press, New York.

Rodriguez, E.K., Hoger, A., and McCulloch, A.D., 1994, "Stress-dependent finite growth in soft elastic tissues," Journal of Biomechanics, Vol. 27, pp. 455-467.

Sah, R.L., Kim, Y.J., Doong, J.Y., Grodzinsky, A.J., Plaas, A.H., and Sandy, J.D., 1989, "Biosynthetic response of cartilage explants to dynamic compression," Journal of Orthopaedic Research, Vol. 7, pp. 619636.

Truesdell, C. and Noll, W., 1965, "The non-linear field theories of mechanics," In: Handbuch der Physik III/3, Ed.: Flügge, S., Springer-Verlag, Berlin.

Williamson, A.K., Chen, A.C., and Sah, R.L., 2000, in review, "Biomechanics of developing articular cartilage," $3^{\text {rd }}$ Symposium of the International Cartilage Repair Society, Vol. 3, 2000. 